\title{
Impact of Transceiver Subsystems on High-Capacity Optical Transmission
}

\author{
L. Galdino, D. Semrau, E. Sillekens, D. Lavery, R. I. Killey and P. Bayvel \\ Optical Networks Group, Dept. of Electronic \& Electrical Engineering, UCL (University College London), UK \\ *p.bayvel@ucl.ac.uk
}

\begin{abstract}
Challenges in the design of transceivers for ultra-high-capacity optical transmission systems are described, together with the details of some approaches to overcome them, description of theoretical models and metrics, and an outlook for the future.
\end{abstract}

\section{Introduction}

The question of how to maximise the overall capacity of optical communications systems has been the subject of much investigation over recent years. The focus has been on optimising the signal-to noise ratio (SNR), as well as the challenges of expanding the usable optical fibre bandwidth [1]. An upper bound on the achievable SNR in coherent optical communication systems is set by the noise introduced by the transceiver subsystems [2]. Significant sources of transceiver noise include quantisation noise due to the finite resolution of digital-to-analog converters (DAC) and analogto-digital converters (ADC) as well as noise from the linear electrical amplifiers. During transmission, the achievable SNR is further limited by amplified spontaneous emission noise (ASE) from optical amplifiers in the transmission line and by nonlinear signal distortion, inherent to transmission through silica optical fibres.

Advances in digital signal processing, modulation formats, high speed electronics, transmission fibres, and broadband optical amplification, have resulted in recent experimental single-mode fibre (SMF) capacity increases. Recent years have seen several landmark transmission demonstrations using single mode fibre, achieving throughputs exceeding $70 \mathrm{Tbit} / \mathrm{s}$ over transatlantic distances [3], with an overall throughput record of $120 \mathrm{Tbit} / \mathrm{s}$ over $630 \mathrm{~km}$ [4]. In this paper we describe the challenges to increasing the signal-to -noise ratio, and therefore data throughput, as well as system design constraints to increasing the optical fibre bandwidth.

\section{Ultra-wideband transmission system design}

Demonstrating ultra-high capacity optical fibre transmission with bandwidths exceeding the conventional and wellestablished EDFA amplifiers is extremely challenging. In addition to the development of new amplifier technologies, it is critically important to design an experimental WDM transmitter to maintain a high optical signal-to-noise ratio (OSNR), as it impacts the signal quality and, therefore, the overall data throughput after transmission. As shown in Fig.1, in an example experimental WDM architecture, the transmitter typically comprises channels under test in an odd and even configuration, which is subsequently coupled to dummy channels or ASE noise source to emulate nonlinear interference noise (NLI) [1] [3] [4] [5]. Therefore, aside from the electrical noise from DACs and ADCs, the highest achievable SNR is further limited by ASE noise introduced by optical amplifiers needed to compensate for optical losses,including those from the modulators (insertion + modulation loss, typically $24 \mathrm{~dB}$ ). A way to enhance the OSNR is to place an optical amplifier before the modulator, however for broadband continuous transmission systems, with bandwidths beyond $\mathrm{C}+\mathrm{L}$

In

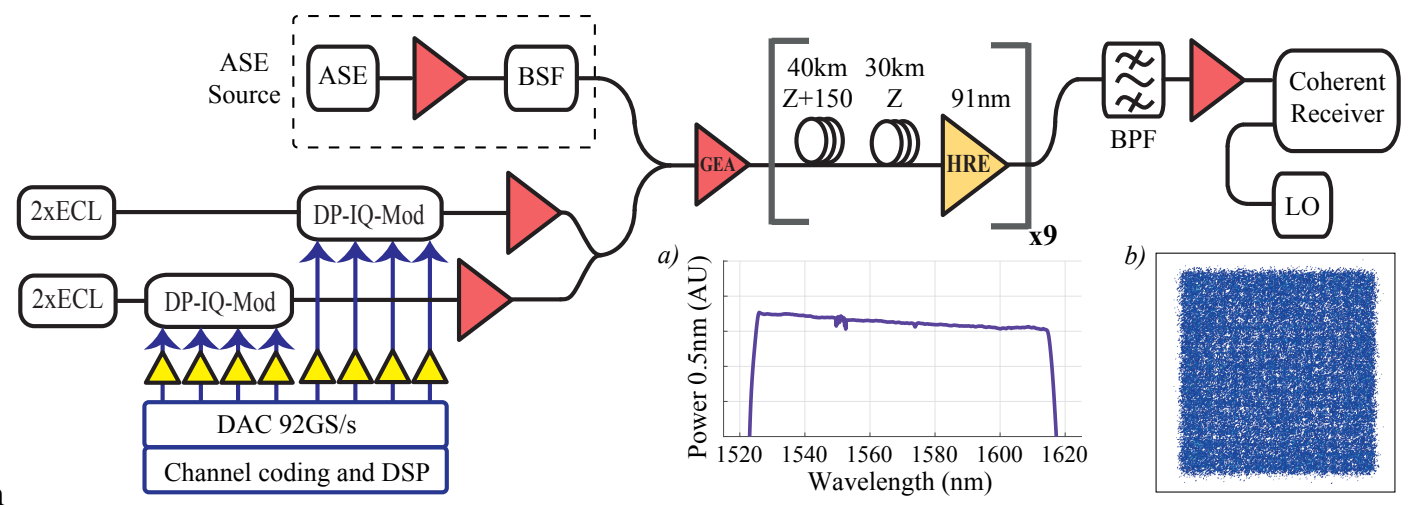

Fig. 1. Schematic of the transmission experiment. Inset a) signal spectrum after the GEA (the modulated channels are centered at $1551 \mathrm{~nm}$. Inset b) 256QAM constellation measured in back-to-back configuration. 

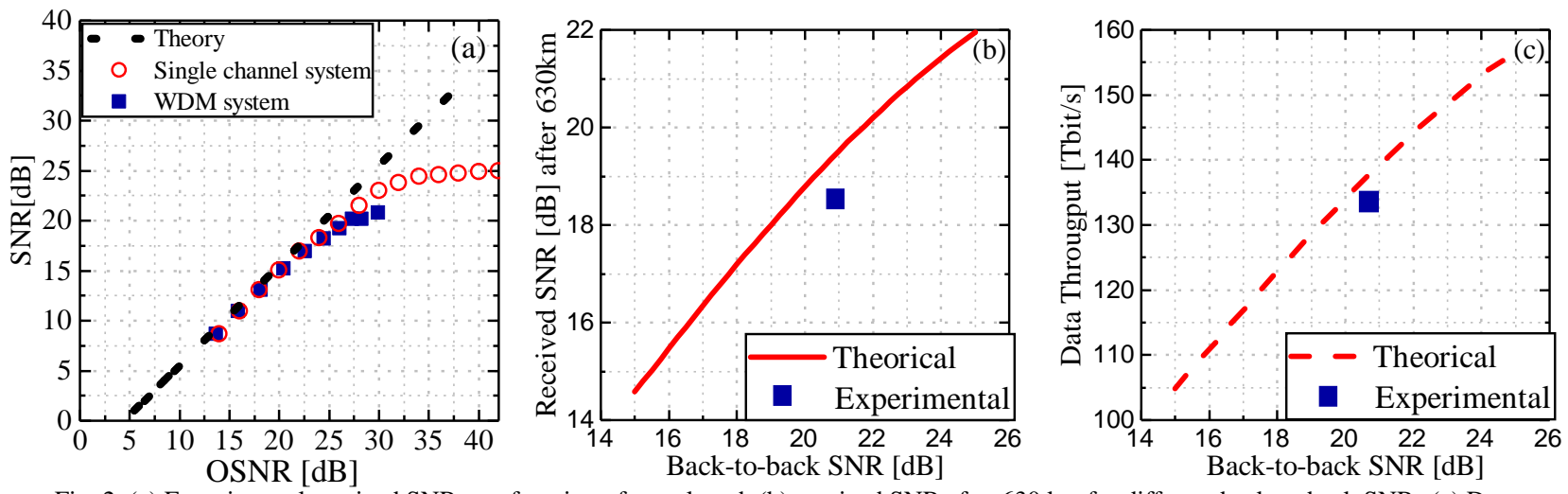

Fig. 2. (a) Experimental received SNR as a function of wavelength (b) received SNR after $630 \mathrm{~km}$ for different back-to-back SNRs (c) Data throughput over $630 \mathrm{~km}$ link for different back-to-back SNRs.

band EDFAs, this is challenging as any other lumped optical amplifier technology is based on nonlinear devices, such as lumped Raman or semiconductor optical amplifier (SOA), which generate nonlinear effects such as four wave mixing and stimulated Brillouin scattering. These are specific challenges in design of a state-of-the-art, advanced and flexible experimental platform - commercial systems would, of course, have independent transceivers.

To assess the constraints of an experimental WDM architecture platform, Fig.2 (a) shows the received SNR as a function of OSNR in a back-to-back (BTB) scenario. The OSNR was measured by adding ASE noise to the signal. The theoretical calculation is shown for performance reference relative to the experimental results of two different experimental transmission platform; single channel 35 GBd PD-256QAM transmitter architecture (laser followed by modulator and an optical amplifier, connected straight to the receiver, detailed setup is described in [2]) and WDM BTB architecture implemented as described in Fig. 1 and in [4]. It is evident that for single channel configuration setup there is a saturation in the highest achievable SNR, dominated by the effective number of bits of the DACs and ADCs, and the noise figure of the electrical drive amplifiers. In the WDM architecture, the OSNR if further degraded to by $11 \mathrm{~dB}$ compared to single channel architecture; from $42 \mathrm{~dB}$ to $31 \mathrm{~dB}$, which causes an reduction of the SNR of $4.2 \mathrm{~dB}$.

To investigate the impact of transceiver constrained SNR of the transmission system performance, experimental and theoretical analysis was carried out using the inter-channel stimulated Raman scattering (ISRS) GN-model [6], with the transmission system modelled using the parameters of the experimental system described in [4]. The plot in Fig. 2 (b) shows the impact of the transceiver-constrained performance on the received SNR after $630 \mathrm{~km}$. This particular experimental system has been well modelled with an offset of the receiver SNR of only $0.4 \mathrm{~dB}$. The model predicts that, as the BTB SNR increases from $16.5 \mathrm{~dB}$ to $25 \mathrm{~dB}$, the received signal SNR after $630 \mathrm{~km}$ transmission increases from $16.5 \mathrm{~dB}$ to $22 \mathrm{~dB}$. For the case of infinite BTB SNR, the model predicts a received signal SNR of $25 \mathrm{~dB}$. It is important to note that after $630 \mathrm{~km}$ of optical fibre transmission the received SNR degraded only $1.9 \mathrm{~dB}$ compared with SNR experimental measured on the BTB configuration.

Finally, to investigate the impact of transceiver constrained SNR in achievable information rate (AIR), Fig. 2 (c) shows the experimental results and theoretical prediction of data throughput as a function of BTB constrained SNR. The mutual information was used as the metric to estimate the AIR. The model predicted a data throughput of $138.2 \mathrm{Tbit} / \mathrm{s}$ for the experimental system under test, compared to $134.4 \mathrm{Tbit} / \mathrm{s}$ experimentally measured. This can be compared with a throughput of 178.6 Tbit/s, ]calculated for the link assuming infinite BTB SNR. Note that, with practical soft-decision decoder, the throughput is further reduced, from $134.4 \mathrm{Tbit} / \mathrm{s}$ to $120 \mathrm{Tbit} / \mathrm{s}$ [4].

For medium- and long-haul transmission links with low noise optical amplifiers, the transceiver-constrained SNR imposes a significant reduction in data throughput in high-capacity ultra-broadband systems. As the transmission distance is increased to ultra-long-haul, the transceiver limited SNR becomes less significant and the system performance becomes dominated by the nonlinear distortion. Future work will focus on longer distance transmission over larger bandwidths with intelligent transceivers capable of sensing and adapting to their environments.

Support from UK EPSRC UNLOC and INSIGHT projects, the Royal Academy of Engineering under the Research Fellowships programme and Xtera is gratefully acknowledged. The authors thank Oclaro for the loan of the modulators

\section{References}

[1] J. Renaudier et al., Proc. ECOC, PDP (2017).

[2] L. Galdino et al., Proc. OFC, W1B.4 (2018).

[3] T-X. Cai et al., Proc. OFC, PDP Th5B.2 (2017).

[4] L. Galdino et al., arXiv:1804.01845 (2018).

[5] J. Cai et al., Proc. OFC, Th1C.1 (2018).

[6] D. Semrau et al., arXiv:1801.02460, (2017) 